\title{
Synthesis and Characterization of Stretchable Ionic Cable
}

\author{
Zubair Ahmed Laghari ${ }^{1}$, Muhammad Ishaque Abro ${ }^{2 a}$, Umair Aftab ${ }^{2 b}$, \\ Razium Ali Soomro ${ }^{3}$, Sajid Hussain Siyal ${ }^{1 b}$
}

RECEIVED ON 24.11.2019, ACCEPTED ON 17.05.2021

\begin{abstract}
A transparent and flexible polymer with unique electric and sound signal transmission capability under highly stretchable conditions has been synthesized. In this paper the milestone achieved in the polymerization of intrinsic ionic $\Omega$-conjugated polymer, hereinafter known as Ionic Cables (ICs), through free radical crosslinking co-polymerization process/technique and structural modifications encountered so far in our experiments are discussed. EIS and stress-strain assessments had showed that ionic conductivity and flexibility are dependent on the amount of accelerator Tetramethylethylenediamine (TEMED) and curing time (CT). By varying the amount of accelerator, in our case TEMED, and CT the ICs with ionic conductivity of $0.021 \mathrm{~S} / \mathrm{cm}$ to $0.111 \mathrm{~S} / \mathrm{cmand} 350 \%$ elongation to $448 \%$ elongation conditions was synthesized with highly water absorption capability up to $\mathbf{7 8 \%}$ of its total volume. The synthesized ICs with ultra-stretchability, transparency and conductivity can be used in versatile applications, including stretchable ionic cables, tissue engineering, ionic mass transport systems and bio sensors.
\end{abstract}

Keywords: Ionic cable, ionic conductivity, elongation, consolidation, ionic crystal, transparent cables

\section{INTRODUCTION}

$\mathrm{I}$ $\mathrm{n}$ most of the cases metallic wires and metallic connectors in electronics are utilized for transfer of electric current and signals. The losses in electronic signal and current followed by circuit heating, rigid in nature and expensive for production are some of the major drawbacks of metal wires and connections [1-3]. Hence, to overcome such issues, the material scientists and engineers are not only engaged in improving conventional electronic components, but also in the race of producing novel materials. The recent openings in the discipline of materials have introduced a new class of polymeric materials with higher conductivity. The estimation of development and characterization of Polymeric Ionic Cables (PICs) has recently attracted scientific community with an effort towards commercialization. The preliminary experimental work had shown that PICs could transmit electrical and sound signals over long distances with enough speed with no variation in current even when stretched eight times of its original length. It is anticipated that ions in PICs are more effective to channel the electrical and audio signals as compared to electrons in metallic electric wires. It is speculated that due to outstanding signal transmitting features, PICs can be used in artificial intelligence, bio sensing functions and electronic conductors with higher stretchability and transparency [4].

The high-water absorbent three-dimensional hydrogel pass through our everyday life in a variety of forms like in the commercial products like shampoo, hair gel, silica gel and other cosmetics, optical lenses,

${ }^{1}$ Department of Metallurgy and Materials Engineering, Dawood University of Engineering and Technology, Karachi, Sindh, Pakistan.Email: ${ }^{a}$ zubair.laghari@ duet.edu.pk, (Corresponding Author), ${ }^{{ }}$sajid.hussain@ duet.edu.pk

${ }^{2}$ Department of Metallurgy and Materials Engineering, Mehran University of Engineering and Technology, Jamshoro, Sindh, Pakistan. Email: ${ }^{a}$ ishaque.abro@ faculty.muet.edu.pk, ${ }^{b}$ umair.aftab@ faculty.muet.edu.pk

${ }^{3}$ Beijing Key Laboratory of Electromechanical Process and Technology for Materials, Beijing University of Chemical Technology, Beijing, China. Email: raziumsoomro@ gmail.com

This is an open access article published by Mehran University of Engineering and Technology, Jamshoro under CC BY 4.0 International License. 
microchips, flexible smart screens etc. This man-made hydrogel is used from general applications to advance cases like sophisticated electronic skin [5, 6], active adaptive lenses [7], stretchable electronics and batteries $[8,9]$.

Long-chain, stretchy polymer can be cross-linked by polymerization techniques with the formation of covalent bonds. A three dimensional network using various monomers, initiators and terminators with the addition of substitutes, the three-dimensional network can absorb the solvent and swells, thus formed material is known as polymer gel, but if water is used as solvent than it would be construed as hydrogel [10]. Various types of polymerization methods are used to make polymers synthetically from which free radical cross-linking co-polymerization of acrylamide is one of the ways for making polymers where molecular weight is built up with slow rate of reaction. It is therefore easy to control variables in the reaction mechanism of polymerization. The functional groups have vital role in holding reactions. Hydrogels are usually prepared by free radical co-polymerization of acrylamide (AAm) based monomers with the chemical cross-linker such as N,N' Methylenebisacrylamide [1$13]$.

The polyacrylamide is a type of hydrogel in which acrylamide is used as a base monomer using thermo initiators, dopants and cross linkers to make a conductive hydrogel with higher flexibility, electrical conductivity and transparency $[14,15]$. The dopants have stable charged sites due to the presence of positive counter ions, where the mobilized ions emerge during doping. In this process free ions move and make hydrogels electrical and sound signal conductors.

The synthesized transparent hydrogel can play a vital role to replace conventional electronic system with flexible and bio compactable electronics. The current and voltage characteristics of conductive hydrogel make an amazing match with high conductive metals like copper and silver.

The current research work presents the formation and characterization of transparent hydrogel which comprises both abilities to stretch and conduct electrical and sound signals with acceptable intensities. The mechanical behavior is also managed to fulfill the engineering factors of safety. The contemporary research will help us to use a capable ionic conductor instead of metallic wires and plug-indisplays in special cases where higher transparency, ionic conductivity and stretchability is required.

\section{EXPERIMENTAL SECTION}

\subsection{Materials and Method}

All chemicals, including acrylamide, lithium chloride (ionic dopants), N,N'-Methylenebisacrylamide (cross linker), ammonium per sulfate (Thermo initiator) and TEMED (accelerator) were obtained from LABCHEM products, BDH Chemicals and Sigma_Aldrich Merck respectively.

Highly water absorbent, conductive, transparent and flexible polyacrylamide base ionic cables (ICs) were synthesized using free radical co-polymerization and cross-linking method. Total of $186 \mathrm{gm}$ solution was prepared using $14 \%$ of acrylamide (monomer), $5 \%$ of lithium chloride (doping agent), $0.043 \%$ of $\mathrm{N}, \mathrm{N}^{\prime}-$ Methylenebisacrylamide (cross linking agent), $0.07 \%$ of ammonium per sulfate (thermo initiator) and TEMED (Thermo initiator) relative to amount of solvent. There were two quantities of TEMED including $0.1 \%$ and $0.2 \%$ used separately to see the effect of TEMED quantity on the characteristics of hydrogel, another factor investigated was CT from 01 to 04 hour(s) applied in muffle furnace. In this practice the TEMED and CT were assorted, samples were labeled as $0.1 / \mathrm{X}$ and $0.2 / \mathrm{X}$, where 0.1 and 0.2 are the amounts of TEMED and $\mathrm{X}$ is $\mathrm{CT}$, after taking distilled water the amount of each chemical is added simultaneously and agitated for 5 minutes at $50^{\circ} \mathrm{C}$ constant curing temperature.

Swelling and de-swelling characteristics under the impact of moisture and temperature was investigated. The shrinkage was measured through weight reduction method where solution was transferred in Petri dish, after drying; it reduced considerable amount of volume in weight reduction as shown in Fig.1.

Volumetric change was calculated using change in initial and final weight of hydrogel after and before 


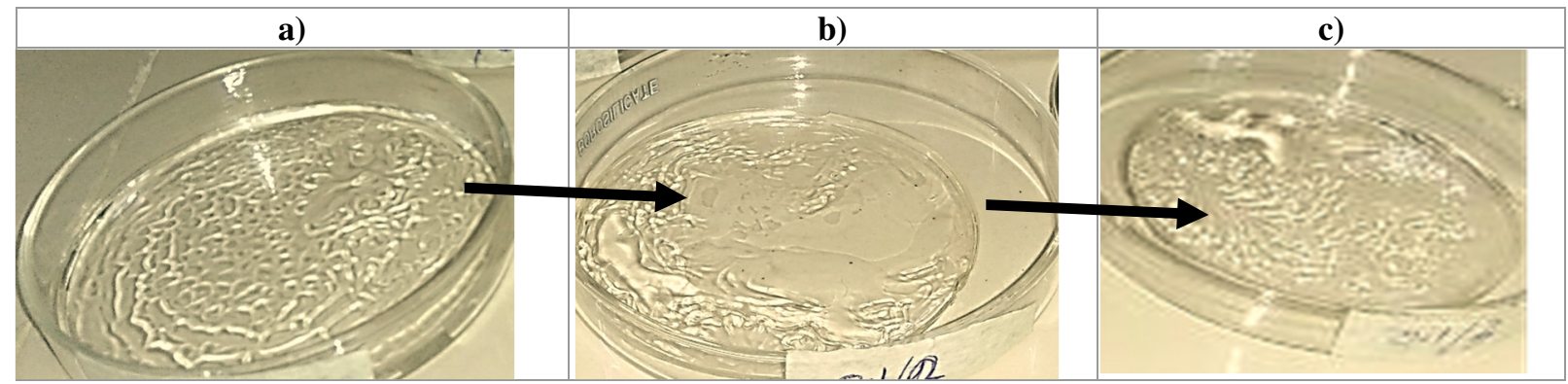

Fig. 1: Volumetric Changes of Hydrogel after (a) One Hour (b) Two Hours and (c) Three Hours Curing

hydrogel curing, using following equation:

$\%$ Weight Reduction $=\frac{\text { Difference in weight }(\mathrm{gm})}{\text { Initial Weight }(\mathrm{gm})} \times 100$

In assessment of the increasing importance, the structure confirmation of polyacrylamide (PAAm) based hydrogels have received considerable attention in the last two decades. In this respect, the vibrational spectroscopy is a potentially useful tool for structural analysis [12]. The IR spectra of synthesized $\mathrm{LiCl}$ doped polyacrylamide gel incorporated in Potassium Bromide pellet was recorded by Fourier Transform Infrared Spectroscopy (FTIR) of Perkin Elmer (Model: Spectrum 2) in the range of 4000-400 $\mathrm{cm}^{-1}$.

The morphology of polyacrylamide hydrogel was investigated using scanning electron microscope (Model: JOEL JSM-6380L) at magnification of $\mathrm{X} 15,000$ and $\mathrm{X} 2,000$ and $20 \mathrm{kV}$. The samples for SEM were prepared by freeze drying followed by sputtercoating with gold to achieve satisfactory results. For mechanical properties, Universal Tensile Machine (Model: ZwickRoell Z005) was used by following ASTM standard D-638 (type V). Electrochemical impedance spectroscopy were carried out using potentiostat (Model: VersaSTAT-500), data was analyzed using Z-view software and bulk resistance was pointed out from those plots at maximum frequencies in real axis.

\section{RESULTS AND DISCUSSION}

\subsection{Volumetric Changes in Hydrogel}

It has been observed that the proportion of TEMED and CT has substantial influence on volumetric changes in ICs. Porosity increment makes ICs more water absorbent and flexible deprived of polymer dissolution in water and keeping ions more activated. Fig.2 ( $\mathrm{X}$ is used as variable for $\mathrm{CT}$ ) shows the volumetric changes of ICs. it is apparent that giving extra CT the samples were dried and permeability minimized during elimination of water, and weight was reduced simultaneously. After increasing the amount of accelerator, the weight reduction rate is recorded much higher than the low amount of TEMED (accelerator). It was due to the higher activation reaction mechanism in polymerization.

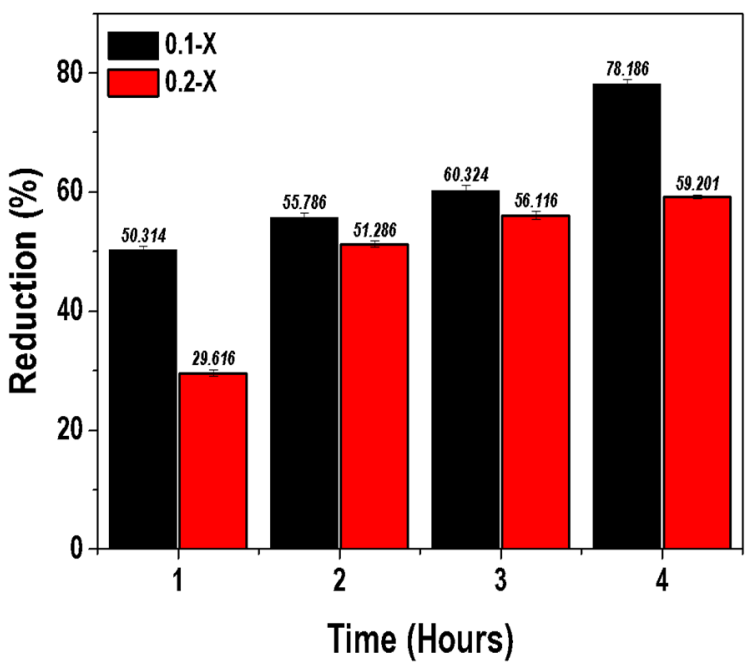

Fig. 2: Reduction of Hydrogel Samples 0.1-1 to 0.1-4 and $0.2-1$ To $0.2-4$

\subsection{Fourier Transform Infrared Spectroscopy of ICs}

The absorption band at $3433 \mathrm{~cm}^{-1}$ shown in Fig. 3 is attributed to the valence vibration corresponding to the $\mathrm{N}-\mathrm{H}$ groups from the cross linking bridges, while the asymmetric valence vibration from $2926 \mathrm{~cm}^{-1}$ is assigned to the $\mathrm{CH}_{2}$ groups included in the 
macromolecular chains and cross linking bridges. The absorption band at $2856 \mathrm{~cm}^{-1}$ is attributed to the $-\mathrm{N}-$ $\mathrm{CH}^{2}$ - bonds from the cross linking bridge, as shown in IR spectra of PAAm in Fig.3. The peak is seen at 1650 $\mathrm{cm}^{-1}$ was a direct result of the amide-I band of the amide group ( $>\mathrm{C}=\mathrm{O}$ stretching vibration frequency). The amide-I band was relocated from $1661 \mathrm{~cm}^{-1}$ in the cross-connected polyacrylamide to $1650 \mathrm{~cm}^{-1}$ [16]. Since $\mathrm{ClO}^{-4}$ is also absorbed at $1100 \mathrm{~cm}^{-1}$, the presence of a strong absorption band at this wavenumber in both PAAm shows that these polymers are doped with this anion. Moreover, the sharp peak around $1650 \mathrm{~cm}-1$ corresponding to $\mathrm{C}=\mathrm{O}$ in the polyacrylamide appears clearly in the copolymer. The conductive properties of polymers are strongly dependent on conjugation length. Defects interrupt conjugation including sp3 carbons, carbonyl groups

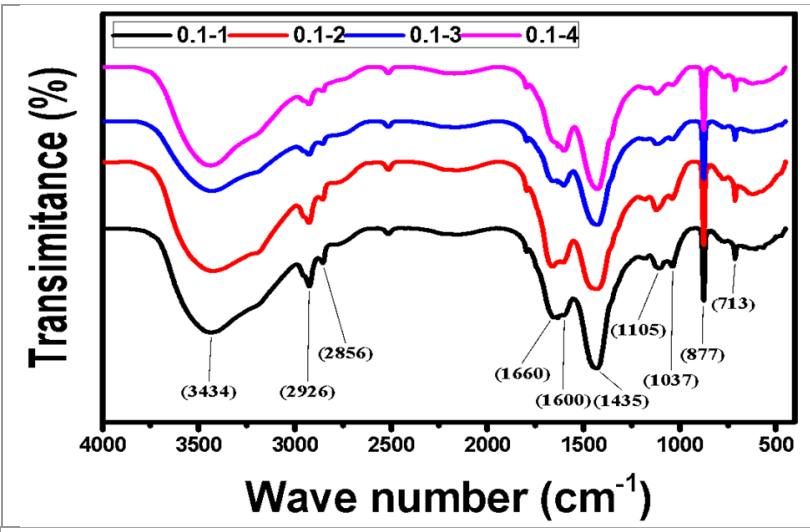

(a) and crosslinks $[17,18]$. Peak of IR spectra at 1095 $\mathrm{cm}^{-1}$ is due to the addition of $\mathrm{LiCl}$ [19].

Peak at $860 \mathrm{~cm}^{-1}$ to $877 \mathrm{~cm}^{-1}$ was stretching of $\mathrm{C}-\mathrm{Cl}$ of alkyl halide group. A weak peak at $713 \mathrm{~cm}^{-1}$ shows the bending of the C-S group which is during ammonium per sulfate used in polymerization.

\subsection{Morphological Studies of ICs}

Structures observed after freeze-drying of the ICs are shown in Fig.4 and Fig.5. the white and bright areas of SEM images are matrices of acrylamide, the intermolecular cross linking is shown through interconnected darker areas, dark areas in SEM images are water possessed porous structure of ICs.

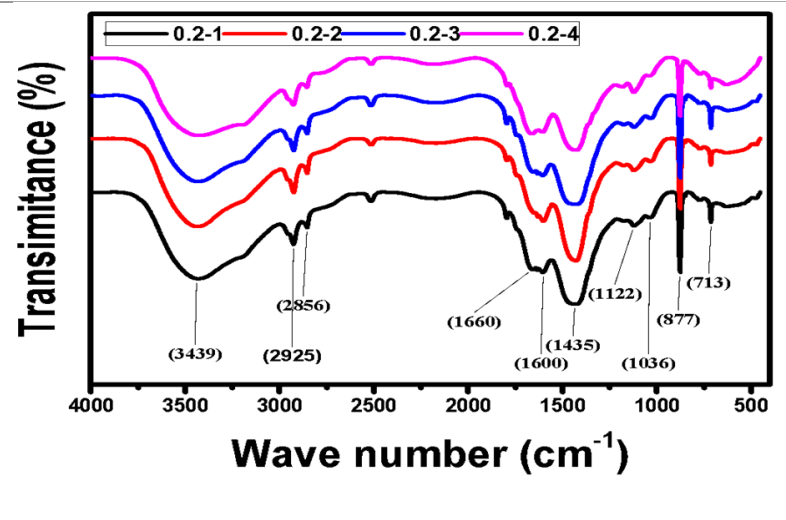

(b)

Fig. 3: FTIR Spectra of Sample a) 0.1/X And Sample b) 0.2/X

a)

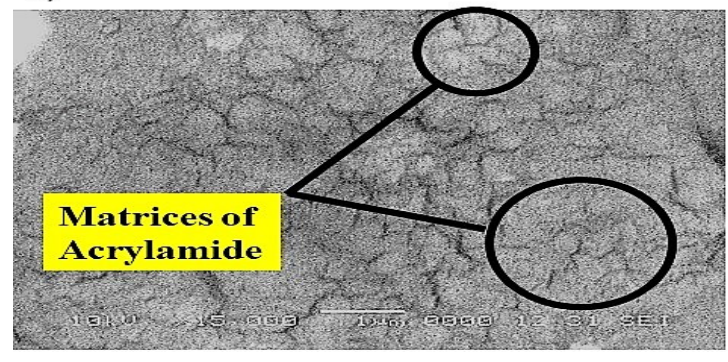

c)

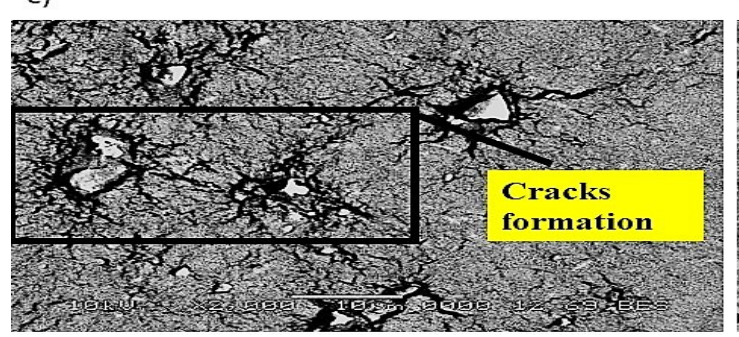

b)

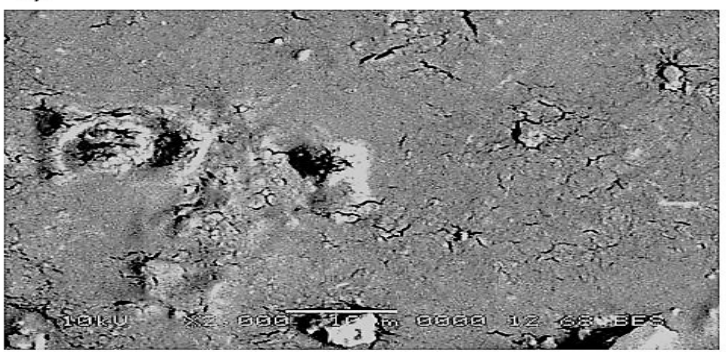

d)

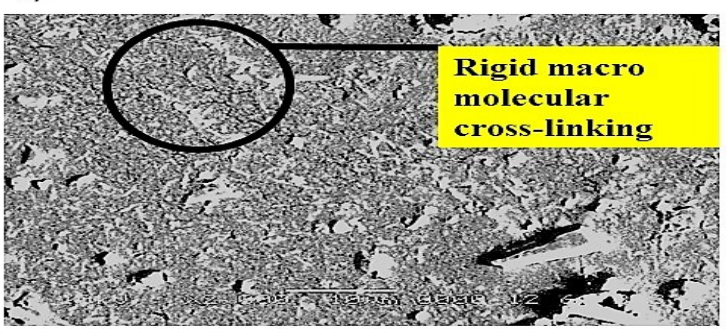

Fig. 4: Scanning Electron Microscopy of Freeze-Dried ICs 0.1/X a) 0.1/1 b) 0.1/2 c) 0.1/3 d) 0.1/4

Mehran University Research Journal of Engineering and Technology, Vol. 41, No. 1, January 2022 [p-ISSN: 0254-7821, e-ISSN: 2413-7219] 
a)

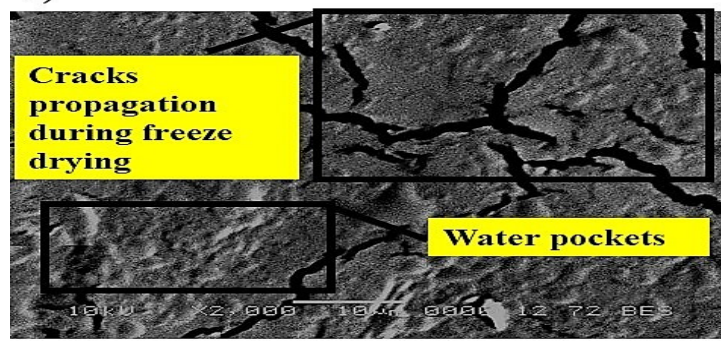

c)

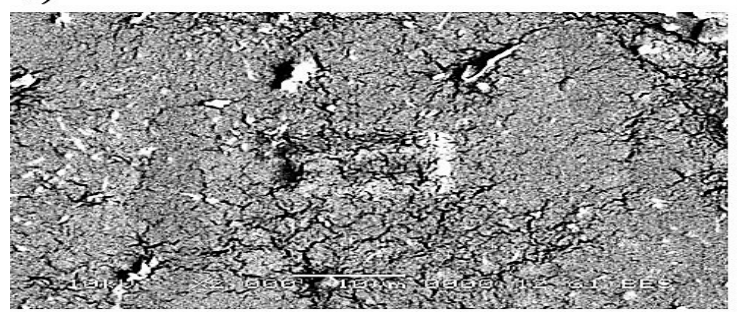

b)

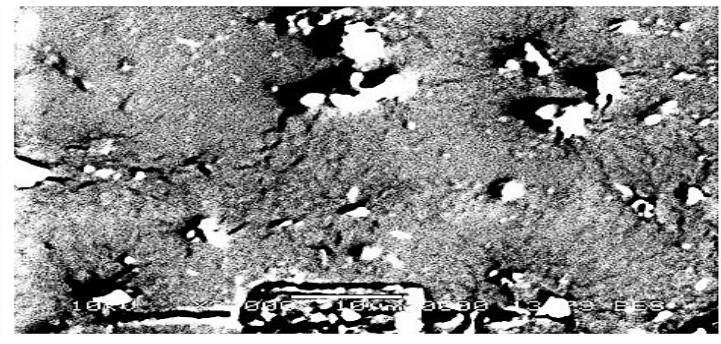

d)

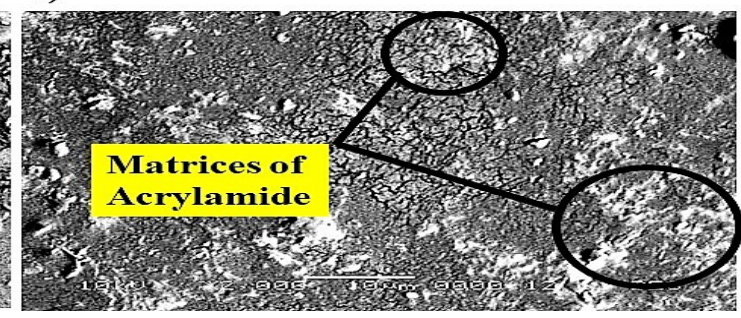

Fig. 5: Scanning Electron Microscopy of Freeze-Dried Hydrogel 0.2/X a) 0.2/1 b) 0.2/2 c) 0.2/3 d) 0.2/4

Fig.4 shows the SEM images of sample 0.1/X which displays the higher concentration of acrylamide matrices than vacant spaces due to the amount of TEMED which accelerate and increases the rate of temperature. The interconnected porous structure is clearly shown in Fig.5. The capillary channels in this observation enable water molecules to inter in hydrogel and make them able to conduct more electricity in the presence of $\mathrm{LiCl}$ ions.

\subsection{Tensile Behavior of ICs}

The large mechanical deformation do not affect the conductivity of ICs, while comparing the tensile strength both the compositions cured at four hours has higher ultimate tensile strength and low ionic conductivity; it is due to the low porosity and removal of condensate water from material, it is also a challenge for materials scientists to maintain both the mechanical properties and electrical properties simultaneously [20], these properties are inversely proportional to each other in ionic conductive hydrogels. It has been observed from the results that increasing the CT in both compositions the UTS and Toughness (area under stress-strain curve) increased. The tensile strength noted was higher in 0.1-4 and 0.24 where maximum water elimination occurred at $50^{\circ} \mathrm{C}$ temperature for four hours as shown in Fig.6.
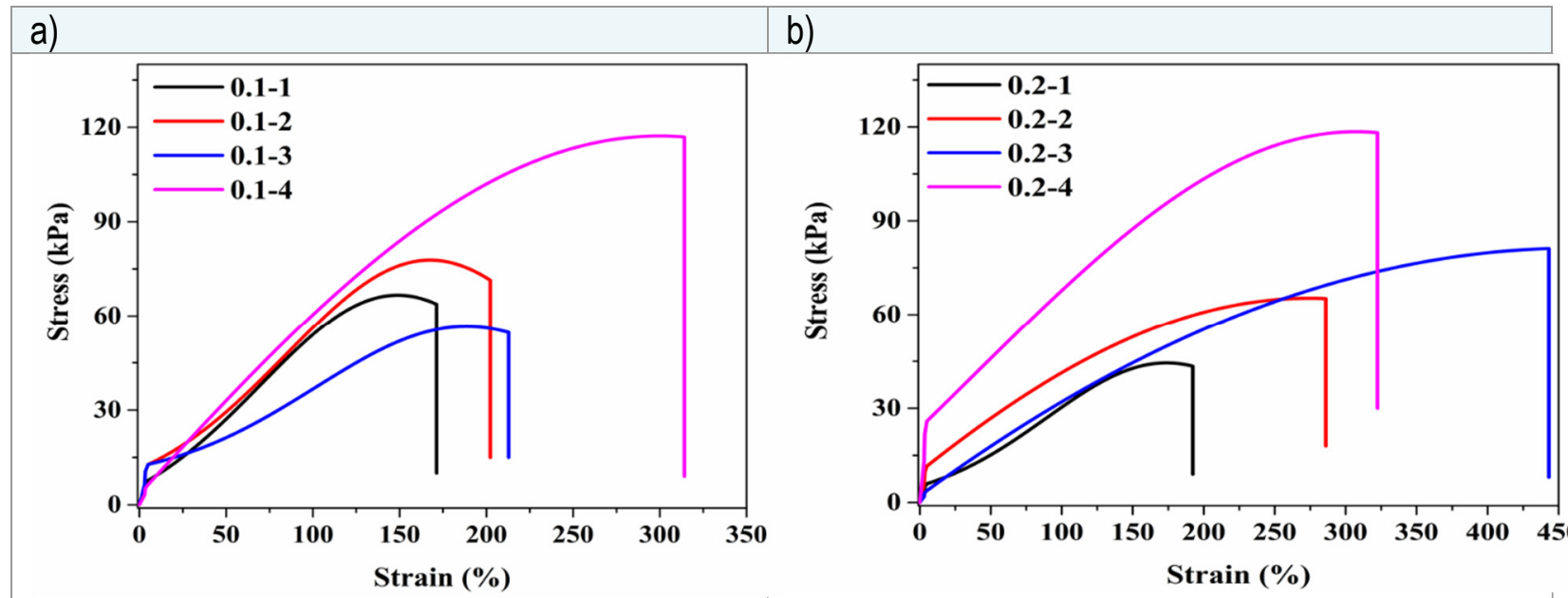

Fig. 6: Stress-Strain Diagram of Polyacrylamide Hydrogel of a) Sample 0.1/X and b) Sample 0.2/X

Mehran University Research Journal of Engineering and Technology, Vol. 41, No. 1, January 2022 [p-ISSN: 0254-7821, e-ISSN: 2413-7219] 
Using $0.1 \%$ of TEMED results $125 \%$ to $325 \%$ strain and $45 \mathrm{kPa}$ to $120 \mathrm{kPa}$ maximum strength shown in Fig.6(a) and by increasing TEMED from $0.1 \%$ to $0.2 \%$ it increases strain from $200 \%$ to $450 \%$ and tensile strength from $30 \mathrm{kPa}$ to $120 \mathrm{kPa}$ as shown in Fig.6(b). The \%age elongation has been noted higher in $0.2 / \mathrm{X}$ than $0.1 / \mathrm{X}$ while equivalent ultimate tensile strength has been recorded. It is also suggested in literature that high friction coefficient in hydrogels comprises more elongation, and in our case this happened when the moisture is removed from hydrogel [21]. It is therefore suggested that the ionic conductivity (See Section 4) and tensile strength could be controlled under diverse environmental conditions.

\section{CONDUCTIVITY MEASUREMENT IN ICs}

Ionic conductivity is subjected to enhance by increasing the amount of water, the amount of accelerator (TEMED) and decreased when decreasing the CT. The bulk resistance was calculated from the Nyquist plots as shown in Fig.7 and the ionic conductivity was calculated using the equation.1. [22]

$\sigma=\frac{\mathrm{t}}{\left(\mathrm{R}_{\mathrm{b}}\right) \mathrm{A}}$

where $R_{b}$ is bulk resistance, $t$ is thickness of the electrolyte and $\mathrm{A}$ is electrode contact area.
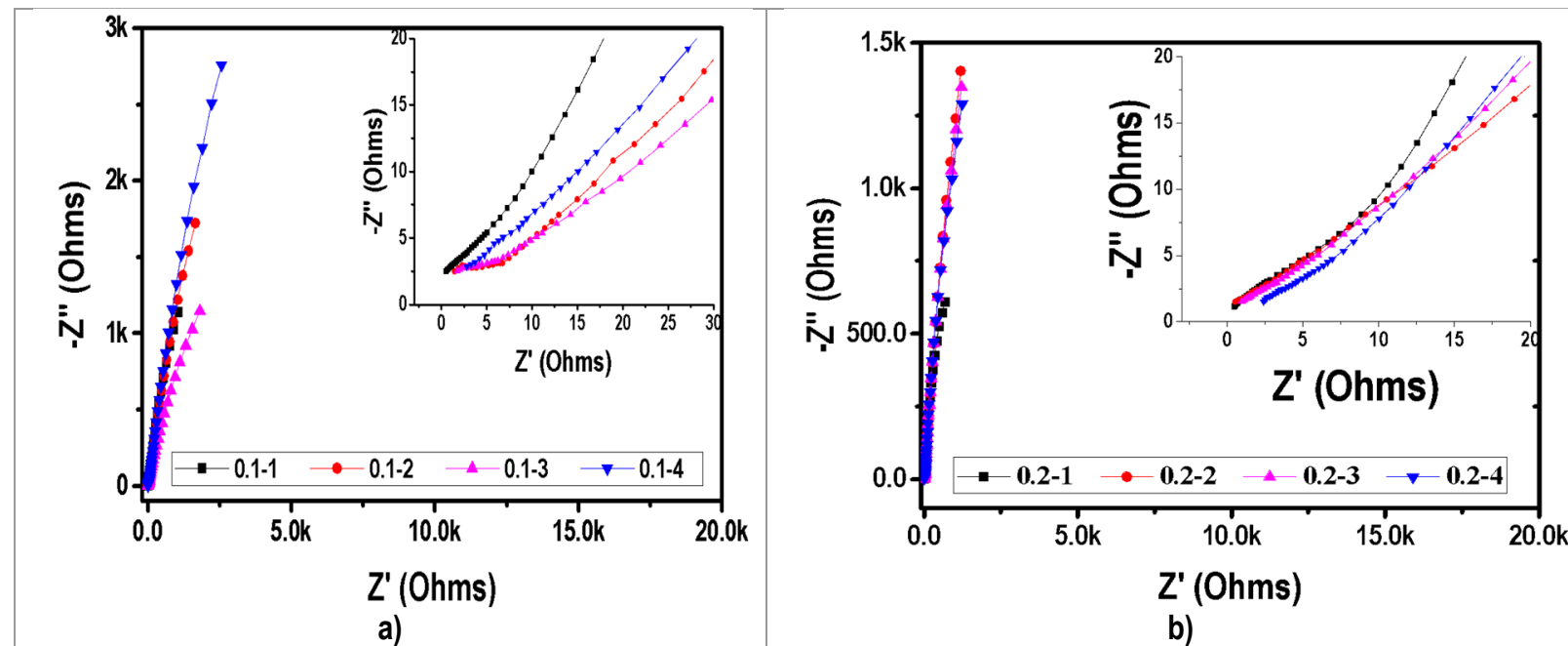

Fig. 7: Nyquist and Bode Plots of ICs of a) Sample 0.1/X and b) Sample 0.2/X

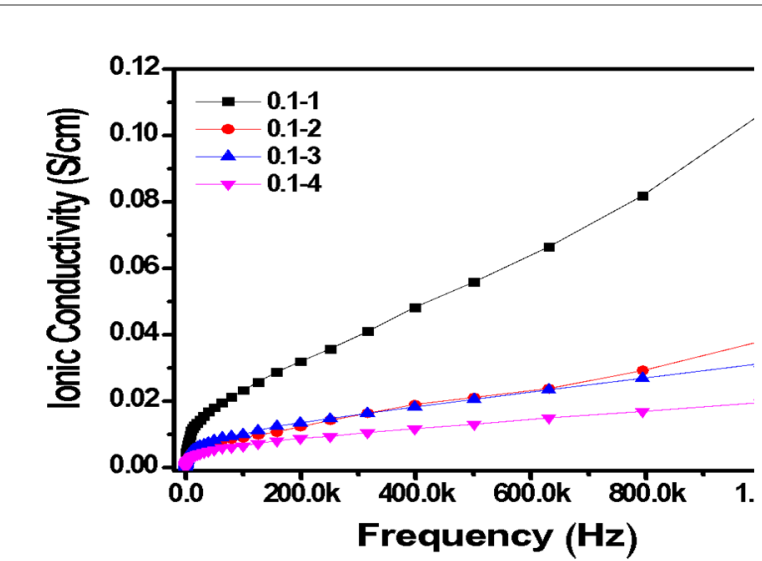

(a)

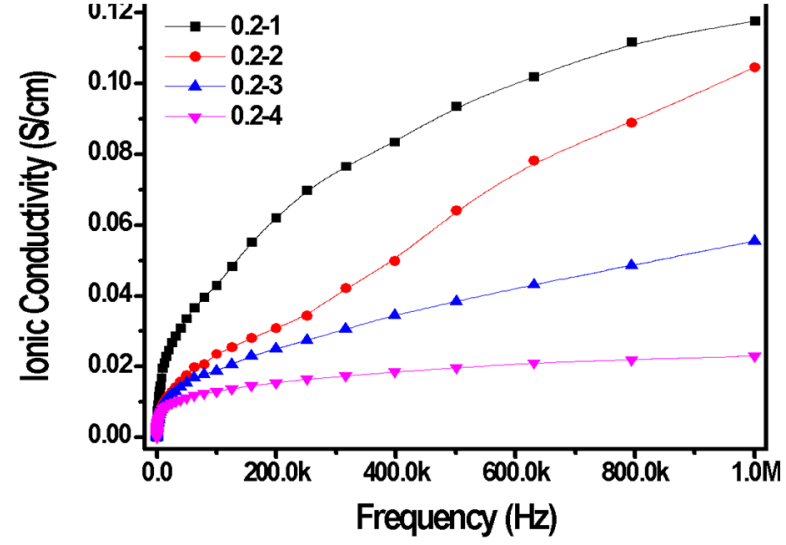

(b)

Fig. 8: Ionic Conductivity of ICs of a) Sample 0.1/X and b) Sample 0.2/X 
From the Nyquist plots the measured ionic conductivity as shown in Fig.8 is further figured out in Table 1 and Table 2 for clear understanding and comparison. It has been noted in given tables that increasing the $\mathrm{CT}$ in both the samples, the ionic conductivity decreases due to the low transport medium and water content, 0.1-1 and 0.2-1 have been observed more ionic conductive than other samples It is observed that moderate level of moisture in polymer structure improves membrane flexibility without much decrease in mechanical strength and ionic conductivity, with increasing the amount of TEMED from 0.1 to 0.2 the conductivity increases, with the comparison of literature the conductivity is achieved in order to $10^{-8}$ in previous reports[23, 24], which is much lower conductivity as compared to our current work rather than in current research.

\begin{tabular}{|c|c|c|}
\hline \multicolumn{3}{|c|}{ Table 1: Ionic conductivity of sample 0.1/X } \\
\hline S.No. & Sample No. & $\begin{array}{c}\text { Ionic Conductivity } \\
(\text { S/cm) }\end{array}$ \\
\hline 1. & $0.1-1$ & 0.106420 \\
\hline 2. & $0.1-2$ & 0.037974 \\
\hline 3. & $0.1-3$ & 0.031414 \\
\hline 4. & $0.1-4$ & 0.019596 \\
\hline
\end{tabular}

\begin{tabular}{|c|c|c|}
\hline \multicolumn{3}{|c|}{ Table 2: Ionic conductivity of sample 0.2/X } \\
\hline S.No. & Sample No. & $\begin{array}{c}\text { Ionic Conductivity } \\
\text { (S/cm) }\end{array}$ \\
\hline 1 & $0.2-1$ & 0.117702 \\
\hline 2 & $0.2-2$ & 0.104624 \\
\hline 3 & $0.2-3$ & 0.055500 \\
\hline 4 & $0.2-4$ & 0.022938 \\
\hline
\end{tabular}

Ionic conductivity measured in this experiment was about $0.02 \mathrm{~S} / \mathrm{cm}$ to $0.11 \mathrm{~S} / \mathrm{cm}$ which is higher than be noted in previous reports[24, 25].

It can also be noted here that the ionic conductivity is much higher in 0.1-1 and 0.1-2 than other samples which witness the higher ionic transportation in hyderogel containing higher water content in porous structure of three-dimensional network.

\section{CONCLUSION}

Following conclusions were drawn from present work:
- The UTM results showed that the strength of ionic cables (ICs) is increasing with increasing the amount of TEMED and curing time (CT).

- EIS results showed that ionic conductivity of ICs is decreasing with the increasing the amount of TEMED.

- The effect of CT on ionic conductivity showed that with increasing $\mathrm{CT}$ ionic conductivity also increased.

- It was noted that ionic conductivity is reciprocal to the UTS and percent elongation features of ICs

- Present study showed that ICs with maximum ionic conductivity $0.11 \mathrm{~S} / \mathrm{cm}$ were obtained when $0.2 \%$ of TEMED was added at CT of 1 hour and strength $120 \mathrm{kPa}$ and percent elongation $450 \%$ was obtained when $0.2 \%$ of TEMED was added. The ionic conductor can perform better in the moist environment under higher ionic mobilization requirement, while under the requirement of higher mechanical strength, the hydrogel must be free or negligible amount of water causes to weaken the bonding among polymer chain.

\section{REFRENCES}

1. Vu M., Ng N. N., Kim M. C., Squire J., "Reducing signal loss in cables", Patent No. US 9,349,507 B2, United States Patent, May 2016.

2. Yang M., Sun Y., Pan X., Wan H., Lu X., "Development and Prospect of Twisted Pair Cables", Proceedings of the IOP Conference Series: Earth and Environmental Science, Vol. 170, No.4, pp. 1-5, 2018.

3. Severengiz M., Sprenger T., Seliger G., "Challenges and Approaches for a Continuous Cable Production", Procedia CIRP, Vol. 40, pp. 18-23, 2016

4. Yang C. H., Chen B., Lu J. J., Yang J. H., Zhou J., Chen Y. M., Suo Z., "Ionic cable," Extreme Mechanics Letters, Vol. 3, pp. 59-65, 2015.

5. Bauer S., "Flexible electronics: sophisticated skin," Nature Materials, Vol. 12, No. 10, p. 871872, 2013.

6. Honda W., Harada S., Arie T., Akita S., Takei K., "Wearable, human-interactive, healthmonitoring, wireless devices fabricated by macroscale printing techniques", Advanced 
Functional Materials, Vol. 24, pp. 3299-3304, 2014.

7. Shian S., Diebold R. M., Clarke D. R., "Tunable lenses using transparent dielectric elastomer actuators", Optics Express, Vol. 21, pp. 86698676, 2013.

8. Fan J. A., Yeo W-H., Su Y., Hattori Y., Lee W., Jung S.-Y., Zhang Y., Liu Z., Cheng H., Falgout L., Bajema M., Coleman T., Gregoire D., Larsen R.J., Huang Y., Rogers J.A., "Fractal design concepts for stretchable electronics", Nature Communications, Vol. 5, p. 3266, 2014.

9. Xu S., Zhang Y., Cho J., Lee J., Huang X., Jia L., Fan J.A., Su Y., Su J., Zhang H., Cheng H., Lu B., Yu C., Chuang C., Kim T.-I., Song T., Shigeta K., Kang S., Dragdeviren C., Petrov I., Braun P.V., Huang Y., Paik U., Rogers J.A., "Stretchable batteries with self-similar serpentine interconnects and integrated wireless recharging systems", Nature communications, Vol. 4, p. 1543, 2013.

10. Ahmed E. M., "Hydrogel: Preparation, characterization, and applications: A review", Journal of Advanced Research, Vol. 6, pp. 105121, 2015.

11. Öztürk M., Coşkun R., Okutan M., Yalçın O., "Origin of the synthetic circuits and the Brownian motion in stretchable crystal violet doped and biocompatible composite hydrogels," Journal of Molecular Liquids, Vol. 249, pp. 211-218, 2018.

12. Tangri A., "polyacrylamide based hydrogels: synthesis, characterization and applications", International Journal of Pharmaceutical, Chemical \& Biological Sciences, Vol. 4, No.4, pp. 951-959, 2014.

13. Okay O., Oppermann W., Polyacrylamide clay nanocomposite hydrogels: Rheological and light scattering characterization," Macromolecules, Vol. 40, pp. 3378-3387, 2007.

14. Bai Y., Chen B., Xiang F., Zhou J., Wang H., Suo Z., "Transparent hydrogel with enhanced water retention capacity by introducing highly hydratable salt", Applied Physics Letters, Vol. 105, p. 151903, 2014.

15. Kim C.-C., Lee H.-H., Oh K. H., Sun J.-Y., "Highly stretchable, transparent ionic touch panel", Science, Vol. 353, pp. 682-687, 2016.

16. Sabbagh F., Khatir N. M., Karim A. K., Omidvar
A., Nazari Z., Jaberi R., "Mechanical Properties and Swelling Behav-ior of Acrylamide Hydrogels using Mont-morillonite and Kaolinite as Clays", Journal of Environmental Treatment Techniques, Vol. 7, pp. 211-219, 2019.

17. Sönmez G., Saraç A. S., "In situ spectroelectrochemistry and colorimetry of poly (pyrrole-acrylamide)s", Journal of Materials Science, Vol. 37, pp. 4609-4614, 2002.

18. Lei J., Cai Z., Martin C. R., "Effect of reagent concentrations used to synthesize polypyrrole on the chemical characteristics and optical and electronic properties of the resulting polymer", Synthetic Metals, Vol. 46, pp. 53-69, 1992.

19. Wieczorek W., Such K., Florjanczyk Z., Stevens J., "Polyether, polyacrylamide, $\mathrm{LiClO} 4$ composite electrolytes with enhanced conductivity," The Journal of Physical Chemistry, Vol. 98, pp. 68406850, 1994.

20. Wei J., Yin C., Wang H., Wang Q., "Polyampholyte-doped aligned polymer hydrogels as anisotropic electrolytes for ultrahigh-capacity supercapacitors", Journal of Materials Chemistry A, Vol. 6, pp. 58-64, 2018.

21. Gong J. P., Katsuyama Y., Kurokawa T., Osada Y., "Double-network hydrogels with extremely high mechanical strength," Advanced Materials, Vol. 15, pp. 1155-1158, 2003.

22. Suganya N., Jaisankar V., Sivakumar E., "Conducting polymeric hydrogel electrolyte based on carboxymethylcellulose and polyacrylamide/polyaniline for supercapacitor applications," International Journal of Nanoscience, Vol. 17, 2018.

23. Ramya C., Selvasekarapandian S., Savitha T., "Proton-conducting membranes: poly ( $\mathrm{N}$-vinyl pyrrolidone) complexes with various ammonium salts," Journal of Solid State Electrochemistry, Vol. 12, pp. 807-814, 2008.

24. Shuhaimi N. E. A., Teo L. P., Woo H. J., Majid S. R., Arof A. K., "Electrical double-layer capacitors with plasticized polymer electrolyte based on methyl cellulose," Polymer Bulletin, Vol. 69, pp. 807-826, 2012.

25. Ganesh B., Kalpana D., Renganathan N. G., "Acrylamide based proton conducting polymer gel electrolyte for electric double layer capacitors", Ionics, Vol. 14, pp. 339-343, 2008. 